\section{Flexible miniature compound lens design for high-resolution optical coherence tomography balloon imaging catheter}

Henry L. Fu, ${ }^{a}$ Yuxin Leng, ${ }^{a}$ Michael J. Cobb, ${ }^{a}$ Kevin Hsu, Joo Ha Hwang, ${ }^{c}$ and Xingde $\mathrm{Li}^{\mathrm{a}, *}$

a University of Washington, Department of Bioengineering, Seattle, Washington 98195

${ }^{\mathrm{b}}$ Micron Optics, Atlanta, Georgia 30345

'University of Washington, Department of Medicine,

Division of Gastroenterology, Seattle, Washington 98195

Abstract. We report on a new optics design for an optical coherence tomography (OCT) balloon imaging catheter. The design involves a miniature compound gradient-index (GRIN) rod lens, which consists of a fiber optic mode-field reducer and relay rod lenses to achieve predictable high lateral resolution at a desired large working distance. The compound lens design significantly simplifies the engineering process for an OCT catheter and enables 3-D full circumferential cross sectional imaging of large luminal organs such as human esophagus. An as-designed OCT catheter is developed and demonstrated for real-time in vivo swine esophagus imaging in a 3-D spiral fashion. ( $) 2008$ Society of Photo-Optical Instrumentation Engineers. [DOI: $10.1117 / 1.3037340]$

Keywords: optical coherence tomography; endomicroscopy; balloon catheter; internal organ imaging.

Paper 08330LR received Sep. 12, 2008; revised manuscript received Oct. 10, 2008; accepted for publication Oct. 14, 2008; published online Dec. 11, 2008.

Optical coherence tomography (OCT) is a rapidly evolving noninvasive imaging technology that provides high-resolution cross sectional images of tissue microanatomy. ${ }^{1}$ While initially applied to eye imaging, the development of miniature fiber optic catheters/endoscopes has enabled high-resolution OCT imaging of internal luminal organs in vivo. Most of the OCT catheters developed so far have a small diameter (e.g., $\sim 1$ to $2 \mathrm{~mm}$ ), a high lateral resolution (e.g., 15 to $40 \mu \mathrm{m}$ ), and a short working distance (e.g., 1 to $4 \mathrm{~mm}$ ). ${ }^{2-6}$ These catheters are well suited for imaging small lumens or for imaging only a small sector of large lumens when the probes are in direct contact with the tissue surface. The short working distance unfortunately precludes full circumferential imaging of large luminal organs such as human esophagus (which has a diameter of $\sim 18$ to $25 \mathrm{~mm}$ when the natural esophageal folds are flattened e.g., by balloon inflation). There is an increasing clinical need for systematic imaging assessment of the entire esophagus for Barrett's esophagus surveillance and early cancer detection, ${ }^{7,8}$ inspiring strong interest in developing a new type of OCT catheter-a balloon imaging catheter, which basically integrates a miniature OCT imaging probe within the

*Tel.: 206-616-4853; Fax: (206) 685-3300. E-mail: xingde@u.washington.edu

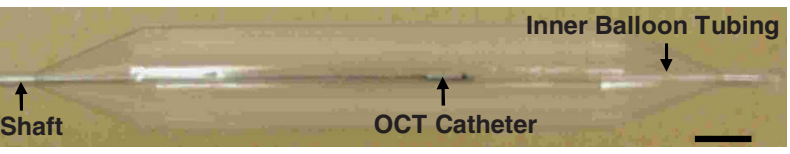

(a)

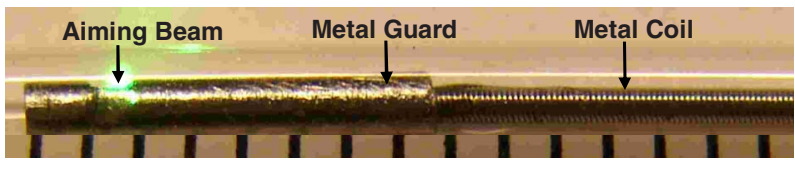

(b)

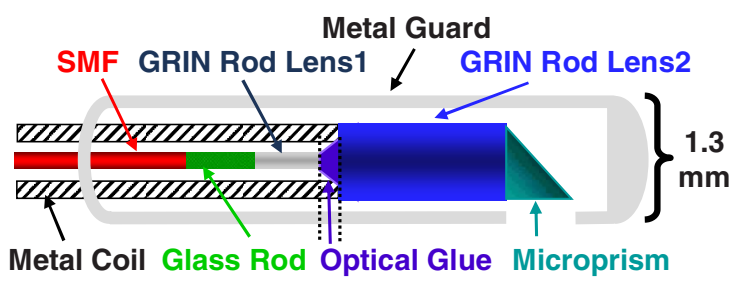

(c)

Fig. 1 (a) Photograph of the OCT imaging catheter inside a balloon that has an $18 \mathrm{~mm}$ diam when inflated. (b) Photograph of the distal end of the OCT imaging catheter. The ruler markings are $1 \mathrm{~mm}$ apart. The catheter including the metal guard has a diameter of $1.3 \mathrm{~mm}$. A visible green aiming beam can be seen projected through the catheter. (c) Schematic of the new distal optics design for the OCT balloon imaging catheter. (Color online only.)

inner lumen of a double-lumen balloon catheter with the balloon (when inflated) to flatten the natural folds of the esophagus. The concept of such an OCT balloon imaging catheter was introduced in 2000 and was recently demonstrated for imaging the entire esophagus. ${ }^{9,10}$ A major challenge with the OCT imaging probe is achieving a small focused spot size (i.e., high transverse resolution) at a large working distance (e.g., 9 to $12 \mathrm{~mm}$ ) while keeping the optical components small (e.g., of a diameter 1 to $2 \mathrm{~mm}$ ), so that the imaging probe would be able to pass through a small inner balloon tubing and shaft, while the entire balloon catheter can be delivered into the esophagus through a 2.8- to 3.4-mm accessory port of a standard gastrointestinal (GI) endoscope. The systematic OCT imaging of the entire esophagus can then be integrated with a routine endoscopy procedure.

We present a new optics design for the OCT balloon imaging catheter. Different from the reported elegant approach where a single lens was formed at the end of a fiber (e.g., by thermal melting) and the engineering conditions were empirically determined for each desired set of resolution and working distance, ${ }^{10}$ the new design utilized an imaging beam mode-field reducer and compound lenses, with the optical parameters well predicted by ray tracing and the engineering process greatly simplified. The OCT balloon catheter consisted of an 18-mm-diam transparent balloon (when inflated) with a 1.4-mm inner lumen [Fig. 1(a)] and a 1.3-mm-diam OCT imaging catheter of a $39-\mu \mathrm{m}$ focused spot size at an $\sim 9$-mm working distance, situated within the inner lumen of the balloon [Figs. 1(a) and 1(b)]. The basic schematic of the new distal end optics design for the imaging catheter is shown

1083-3668/2008/13(6)/060502/3/\$25.00 @ 2008 SPIE 
in Fig. 1(c). The design utilized a microcompound lens made of a glass rod, and two gradient-index (GRIN) rod lenses (uncoated). The glass rod and the first GRIN rod lens formed a spot size reducer, which tightly focused the beam from the single-mode fiber (SMF) down to a size smaller than the mode field diameter of the SMF. This first focused spot was then relayed to the sample by the second GRIN rod lens with a lager diameter (e.g., $1.0 \mathrm{~mm}$ ). The beam would diverge at a much faster rate after the first GRIN rod lens due to the reduced spot size, and thus a larger numerical aperture of $\sim 0.5$ was used for the second GRIN rod lens.

To facilitate the engineering process, the glass rod and the first GRIN rod lens were chosen to have the same diameter as an SMF (i.e., $125 \mu \mathrm{m}$ ), permitting these components to be robustly connected one after another using thermal fusion. For a 0.3 -pitch 125 - $\mu \mathrm{m}$ GRIN rod lens (the first GRIN lens), the length of the glass $\operatorname{rod}(\sim 0.3 \mathrm{~mm})$ that connected the SMF and the first GRIN lens must be carefully selected to achieve the smallest focused spot size at the exit surface of the first GRIN lens. This spot size was estimated to be $3.5 \mu \mathrm{m}$ by ray tracing, representing a significant reduction from the original SMF core size (i.e., $9.3 \mu \mathrm{m}$ ). The final spot size and working distance were controlled by the spacer gap between the first and second GRIN rod lenses [see Fig. 1(c)]. This gap $(\sim 150 \mu \mathrm{m})$ was fine tuned during the catheter assembling process so that the desired working distance was achieved $(\sim 9 \mathrm{~mm})$. The small gap between the two GRIN rod lenses permitted use of optical cement to glue together the two lenses of different diameters $(125 \mu \mathrm{m}$ and $1 \mathrm{~mm}$, respectively), which is similar to the procedure used in engineering a traditional OCT imaging catheter of a single lens and a short working distance. ${ }^{9}$ When used in series, these components at the distal end of the catheter formed a microcompound lens that allowed the catheter to achieve our desired working distance, while maintaining a reasonably small focused spot size, a small overall diameter, and a very simple, robust engineering process. The microprism (with the hypotenuse coated with aluminum) was the last optical component that was glued onto the second GRIN lens to redirect the beam 90 deg toward the tissue. The entire distal end optics of the catheter was then encased in a metal guard for protection. A hollow metal coil of a $0.9-\mathrm{mm}$ outer diameter was used to house the SMF that ran from the proximal to the distal end of the imaging catheter and to transfer the torque for circular imaging. The measured parameters of the catheter were a working distance of $9.6 \mathrm{~mm}$ and a spot size of $39 \mu \mathrm{m}$, very close to the target values predicted by ray tracing (i.e., 9.0-mm working distance and $34.5-\mu \mathrm{m}$ focused spot size). The outer diameter of the imaging catheter including the protection guard was approximately $1.3 \mathrm{~mm}$, which fitted easily into the inner lumen of the balloon. The proximal end of the catheter was connected to a fiber optic rotary joint [not shown in Fig. 1(c)] which functioned as the catheter scanning mechanism. The rotary joint was then placed on a computer-controlled, handheld linear translation stage with micrometer precision. A 3-D spiral beam scanning pattern was created by pulling back the rotary joint (along with the imaging catheter within a stationary balloon) while the joint was rotating, which is similar to the pullback operation of an intravascular ultrasound (IVUS) catheter. $^{11}$

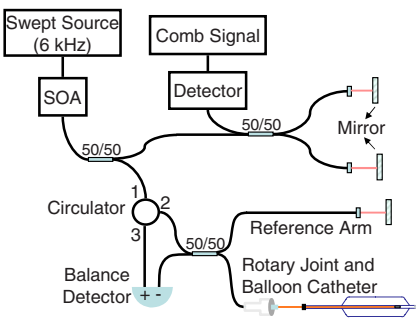

(a)

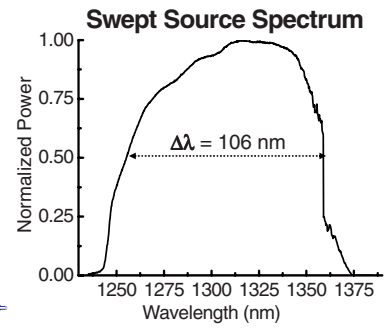

(b)
Fig. 2 Overview of an SSOCT system. (a) Schematic of the SSOCT system used for in vivo imaging with the OCT balloon imaging catheter. (b) Optical spectrum of the swept source.

Preliminary real-time OCT imaging with the balloon catheter was experimentally demonstrated using a swept source OCT system (SSOCT) [Fig. 2(a)] where a frequency swept light source was employed with a center wavelength of $1310 \mathrm{~nm}$, a full width half maximum (FWHM) bandwidth of $\sim 106 \mathrm{~nm}$, and a sweep rate of $6 \mathrm{kHz}$ (corresponding to 12,000 axial scans/sec). The light from the source was amplified to a total power of $\sim 25 \mathrm{~mW}$ using a semiconductor optical amplifier (SOA). ${ }^{12}$ A portion $(\sim 5 \%)$ of the source light from the SOA was sent to a stand-alone interferometer that had an optical path length offset between its two arms roughly equal to the designed imaging depth (i.e., $\sim 2.4 \mathrm{~mm}$ ) to produce optical comb signals for triggering data acquisition. The remaining light was coupled to the OCT setup, which utilized a balanced detection scheme. The incident power at the tissue was approximately $4 \mathrm{~mW}$. The optical spectrum after the SOA is shown in Fig. 2(b). Since the SOA was operating at its saturation current, the powers of forward and backward frequency sweeps were approximately equal. At $6-\mathrm{kHz}$ sweep rate, full-circumferential images were acquired (by the balloon imaging catheter), processed, displayed, and saved in real time at 4 frames/sec with each frame consisting of $2000 \times 3000$ pixels (axial $\times$ circumferential). The measured resolution of the imaging system was $12 \mu \mathrm{m}$ (axial) and $39 \mu \mathrm{m}$ (circumferential), with a detection sensitivity of $\sim 110 \mathrm{~dB}$ (at a 4-mW incident power on the sample).

In vivo full-circumferential $3-\mathrm{D}$ imaging of pig esophagus was conducted with the balloon imaging catheter along with the SSOCT system. The animal was sedated and an overtube was inserted into the upper esophagus. The deflated balloon was passed through the overtube and into the esophagus. An endoscope was then passed through the overtube behind the balloon to monitor the balloon position. A 2-cm-long segment of the esophagus was imaged using the spiral scanning pattern (rotation plus pullback) with $20-\mu \mathrm{m}$ spacing between adjacent circumferential frames. The entire imaging took $\sim 4 \mathrm{~min}$. A real-time 3-D spiral imaging movie is shown in Video 1. A representative 2-D cross sectional image (snapshot) is displayed in Fig. 3(a). The image clearly shows that the balloon catheter, in conjunction with the SSOCT system, is capable of delineating the normal layered structures of the esophagus in vivo, including the epithelium, lamina propria, muscularis mucosae, submucosae, and muscularis propria. The reconstructed 3-D image from the series circumferential images with a $20-\mu \mathrm{m}$ pitch is shown in Fig. 3(b). These initial results demonstrate the ability of the as-designed OCT balloon imaging 


\section{JBO LETTERS}

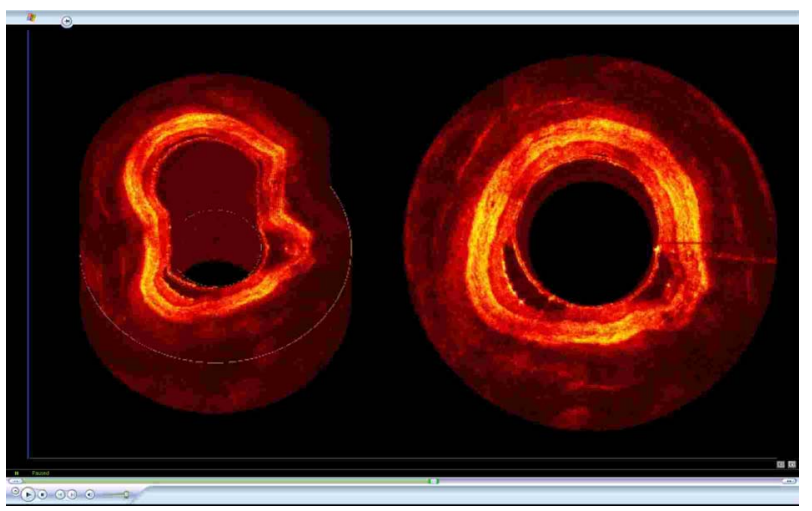

Video 1. Imaging of a 2-cm long segment of the esophagus using the spiral scanning pattern, rotation plus pullback, with $20-\mu \mathrm{m}$ spacing between adjacent circumferential frames. (QuickTime, 3.1 MB). [URL: http://dx.doi.org/10.1117/1.3037340.1].

catheter to generate full-circumferential cross sectional images over a significant longitudinal length of the esophagus with a high resolution.

In summary, we have demonstrated a new design for an OCT balloon imaging catheter that is based on the concept of microcompound lenses. The new design eases the engineering process and is capable of achieving small focused spot size at a large working distance in a predictable fashion. An in-vivo animal model study was performed, and the results demonstrated the feasibility of the as-designed balloon imaging catheter for high-resolution 3-D esophageal imaging, suggesting
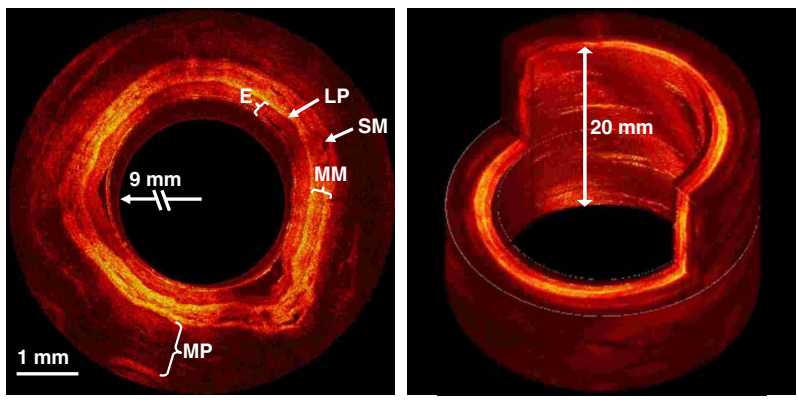

(a)

(b)

Fig. 3 (a) Representative 2-D OCT image $(2000 \times 3000$ pixels, axial $\times$ circumferential) of pig esophagus acquired in vivo with the balloon imaging catheter. Different stratified layers of the esophagus can be clearly identified, including epithelium (E), lamina propria $(\mathrm{LP})$, muscularis mucosae (SM), submucosae (SM), and muscularis propria (MP). (b) A more comprehensive 3-D image constructed from the series of spiral scans of the esophagus. The longitudinal scan distance is $20 \mathrm{~mm}$. the potential of the microcompound lens-based OCT catheter for systematic imaging of human esophagus in clinics.

\section{Acknowledgment}

The authors are grateful to Jiefeng Xi, Daniel J. MacDonald, and Jeff Magula for their assistance with the imaging system. We also thank Addie Warsen, Frank Starr, and Cindy Warren for their assistance in tissue collection and the in-vivo experiments. This work was partially supported by the National Institutes of Health (R21 CA116442, R01 CA120480), the National Science Foundation (Career Award, Li) and Coulter Foundation Translational Research Awards (Li and Hwang).

\section{References}

1. D. Huang, E. A. Swanson, C. P. Lin, J. S. Schuman, W. G. Stinson, W. Chang, M. R. Hee, T. Flotte, K. Gregory, C. A. Puliafito, and J. G. Fujimoto, "Optical coherence tomography," Science 254(5035), 1178-1181 (1991).

2. G. J. Tearney, S. A. Boppart, B. E. Bouma, M. E. Brezinski, N. J. Weissman, J. F. Southern, and J. G. Fujimoto, "Scanning single-mode fiber optic catheter-endoscope for optical coherence tomography," Opt. Lett. 21(7), 543-545 (1996).

3. A. M. Rollins, R. Ung-arunyawee, A. Chak, R. C. K. Wong, K. Kobayashi, M. V. Sivak, and J. A. Izatt, "Real-time in vivo imaging of human gastrointestinal ultrastructure by use of endoscopic optical coherence tomography with a novel efficient interferometer design," Opt. Lett. 24(19), 1358-1360 (1999).

4. X. M. Liu, M. J. Cobb, Y. Chen, M. B. Kimmey, and X. D. Li, "Rapid-scanning forward-imaging miniature endoscope for real-time optical coherence tomography," Opt. Lett. 29(15), 1763-1765 (2004).

5. A. R. Tumlinson, B. Povazay, L. P. Hariri, J. McNally, A. Unterhuber, B. Hermann, H. Sattmann, W. Drexler, and J. K. Barton, "In vivo ultrahigh-resolution optical coherence tomography of mouse colon with an achromatized endoscope," J. Biomed. Opt. 11, 064003 (2006).

6. D. Wang, B. V. Hunter, M. J. Cobb, and X. D. Li, "Super-achromatic rapid scanning microendoscope for ultrahigh-resolution OCT imaging," IEEE J. Sel. Top. Quantum Electron. 13(6), 1596-1601 (2007).

7. M. I. Canto,"Barrett's esophagus," Gastrointest Endosc Clin. N. Am. 15(1), 83-92 (2005).

8. K. K. Wang, "Current strategies in the management of Barrett's esophagus," Curr. Gastroen. Rep. 7(3), 196-201 (2005).

9. X. D. Li, S. A. Boppart, J. Van Dam, H. Mashimo, M. Mutinga, W. Drexler, M. Klein, C. Pitris, M. L. Krinsky, M. E. Brezinski, and J. G. Fujimoto, "Optical coherence tomography: advanced technology for the endoscopic imaging of Barrett's esophagus," Endoscopy 32(12), 921-30 (2000).

10. B. J. Vakoc, M. Shishko, S. H. Yun, W. Y. Oh, M. J. Suter, A. E. Desjardins, J. A. Evans, N. S. Nishioka, G. J. Tearney, and B. E. Bouma, "Comprehensive esophageal microscopy by using optical frequency-domain imaging (with video)," Gastrointest. Endosc. 65(6), 898-905 (2007).

11. J. M. Tobis, J. Mallery, D. Mahon, K. Lehmann, P. Zalesky, J. Griffith, J. Gessert, M. Moriuchi, M. McRae, M. L. Dwyer, and et al., "Intravascular ultrasound imaging of human coronary arteries in vivo. Analysis of tissue characterizations with comparison to in vitro histological specimens," Circulation 83(3), 913-26 (1991).

12. R. Huber, M. Wojtkowski, K. Taira, J. G. Fujimoto, and K. Hsu, "Amplified, frequency swept lasers for frequency domain reflectometry and OCT imaging: design and scaling principles," Opt. Express 13(9), 3513-3528 (2005). 